\title{
Emerging Therapies for the Treatment of Psoriasis
}

\author{
Mahir Patel • Antoinette Day • Richard B. Warren • Alan Menter
}

To view enhanced content go to www.dermtherapy-open.com

Received: July 30, 2012 / Published online: October 25, 2012

(C) The Author(s) 2012. This article is published with open access at Springerlink.com

\section{ABSTRACT}

Psoriasis is an immune-mediated disease that affects $1 \%-2 \%$ of the European and North American population. While topical agents such as corticosteroids and vitamin D derivatives are prescribed for mild disease, they are generally unable to adequately control patients with more severe disease. Over the past decade, research into the immunopathogenesis of psoriasis, including

M. Patel $(\bowtie) \cdot$ A. Menter

Baylor Research Institute, Baylor University Medical Center, 3900 Junius Street Suite 125, Dallas,

TX 75246, USA

e-mail: mahir83@gmail.com

A. Day

College of Medicine, Texas A\&M Health Science

Center, 8447 State Hwy 47, Bryan, TX 77807, USA

R. B. Warren

The Dermatology Centre, Manchester Academic Health Science Centre, Salford Royal NHS Foundation Trust, The University of Manchester, Manchester M6 8HD, UK

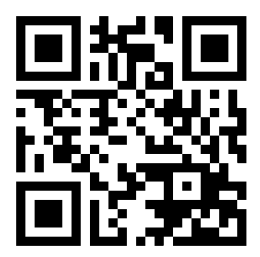

Enhanced content for this article is available on the journal web site: www.dermtherapy-open.com investigations into the role of tumor necrosis factor-alpha and more recently interleukins (IL) $12 / 23$, has led to the advent of targeted biologic therapies based on the central role of a new subset of T cells, Th17. Because of their increased specificity, biologic agents have revolutionized short- to medium-term treatment outcomes and safety profiles for moderate to severe disease over previously gold standard systemic agents. The immunopathogenesis of the disease is still a focus for researchers and novel targets for future agents are being discovered and investigated in clinical trials. In particular, specifically targeting the IL-23/Th17 pathway has given rise to IL23p19 and IL-17 antagonists, both of which have shown significant promise in clinical trials. IL-22 is involved in keratinocyte proliferation and is being studied as a treatment target for psoriasis. New small molecule oral agents, including Janus kinase and phosphodiesterase inhibitors are currently in phase 2 and 3 clinical trials.

Keywords: Biologic therapies; Interleukin-12/ 23; Interleukin-17; Interleukin-20/22; Janus kinase inhibitors; Phosphodiesterase 4 inhibitors; Psoriasis 


\section{INTRODUCTION}

Psoriasis is an inflammatory disease that affects $1-2 \%$ of the European and North American populations [1]. It typically presents with erythematous, scaly, raised, well-demarcated lesions on the scalp, trunk, and extensor surfaces; however, any body site can be involved. Up to $30 \%$ of patients suffer from an associated arthritis, known as psoriatic arthritis [2], and patients with more extensive disease have diminished quality of life because of their skin and joint disease [3]. Furthermore, there are genetic links with conditions such as Crohn's disease as well as associations with obesity, type 2 diabetes, and cardiovascular mortality. Current work is attempting to establish if psoriasis is a specific risk factor per se for these conditions or if factors such as increased smoking and alcohol use in patients with psoriasis accounts for such associations $[4,5]$.

Typically, topical agents are first-line therapy for patients with mild psoriasis but generally ineffective for patients with more severe disease where a range of therapies, including phototherapy, systemic, and/or biological agents are often used. Current gold standard biologic therapies targeting anti-tumor necrosis factor (TNF) alpha and anti-interleukin-12p40 (anti-IL-12p40) have dramatically improved clinical outcomes in patients with psoriasis with improved short-term safety profiles (i.e., less organ specific toxicity) compared to widely used systemic agents. Despite this improvement, there are subpopulations of patients that are either nonresponders to currently available biological agents and/or have experienced diminishing therapeutic benefit over time [6, 7]. Moreover, current biologic agents still have the potential to cause significant side effects in a subset of patients with their longer-term safety yet to be fully evaluated [8]

Therefore, researchers continue to try and further elucidate the immunopathogenesis of psoriasis in an attempt to develop new therapeutic agents, including newer oral agents, a method of administration preferred by some patients. The purpose of this article is to review new therapeutic avenues, including anti-IL23, IL-17, and IL-22 antagonists and oral agents, including Janus kinase (JAK) and phosphodiesterase 4 (PDE4) inhibitors.

\section{METHODS}

A search for phase 1-3 clinical trials for treatment of moderate to severe chronic plaque psoriasis using agents targeting IL-23, IL-17, and IL-22 antagonists and oral agents, including JAK and PDE4 inhibitors, was performed in a nonsystematic fashion. Eligible participants with moderate to severe chronic plaque psoriasis typically include patients with $\geq 10 \%$ Body Surface Area (BSA), $\geq 12$ Psoriasis Area and Severity Index (PASI) and are candidates for phototherapy and/or systemic therapy. Patients with non-plaque forms of psoriasis were excluded.

\section{TUMOR NECROSIS FACTOR ALPHA INHIBITORS}

The TNF-alpha pathway is an established target for psoriasis and psoriatic arthritis therapies, with current licensed anti-TNF medications including infliximab, adalimumab, and etanercept. Anti-TNF agents have been used to treat over 2 million patients with immunemediated diseases for extended durations. Thus its use in psoriasis has been well studied and new agents are still being developed. Certolizumab-pegol is a fragment antigen 
binding segment of an anti-TNF antibody and pegylated to allow for a longer half-life. In phase 2 clinical trials for the treatment of psoriasis, patients randomized to $400 \mathrm{mg}$ every other week and $200 \mathrm{mg}$ every other week showed PASI 75 response in $82.8 \%$ and $74.6 \%$ of patients respectively [9]. Golimumab, a newer anti-TNF therapy, with a license for psoriatic arthritis, does show efficacy in the treatment of psoriasis. Phase 3 data demonstrated PASI 75 response in patients receiving 50 or $100 \mathrm{mg}$ of golimumab every 4 weeks. PASI 75 response in 50 and $100 \mathrm{mg}$ were seen in $40 \%$ and $58 \%$ respectively compared to $3 \%$ in placebo at week 14 and $56 \%$ and $66 \%$ respectively, compared to $1 \%$ of placebo group seen at week 24 [10]. ART621, a low molecular weight anti-TNF agent, consists of two identical antibodies and is believed to have improved tissue penetration. In a phase 2 study for treatment in plaque psoriasis, four subjects showed a PASI 50 response in a 12-week treatment period compared to zero in the placebo arm [11].

\section{INTERLEUKINS 12 AND 23}

IL-23 is a heterodimer consisting of a p19 and a p40 subunit. IL-23 regulates Th17 CD4 cells, which produce IL-17, a proinflammatory cytokine [12]. IL-12 is a heterodimer containing a p35 and p40 subunit. Production of IL-12 by phagocytes and dendritic cells links innate and adaptive immunity and activates signal transducer and activator of transcription 4 (STAT4), which increases the production of interferon gamma [1]. Studies have shown that in psoriatic lesions there is an overexpression of both IL-12 and IL-23. Many of these studies, however, used the presence of the shared p40 subunit to measure the expression of IL-12 and thus were unable to differentiate between the presence of IL-12 and IL-23 [13-16].
Ustekinumab is a human immunoglobulin G1kappa monoclonal antibody to the p40 subunit, and therefore inhibits the actions of IL-12 and IL-23 (Table 1) [9-11, 17-22]. Phase 2 randomized controlled trials of 146 patients with psoriatic arthritis showed that $42 \%$ of subjects achieved an American College of Rheumatology-20 response at 12 weeks (Table 1), whereas only $14 \%$ of placebo-treated patients achieved similar results [17]. Phase 3 trials for psoriatic arthritis are ongoing. In randomized phase 3 trials involving 1,966 patients with psoriasis, $74 \%-77 \%$ of patients achieved a PASI 75 response after 12 weeks (Table 1) [23]. During these trials, $55 \%$ of patients experienced one or more adverse event compared to $50 \%$ of patients dosed with placebo [23]. Major adverse cardiovascular events (MACE) were also reported during these trials, predominantly in the initial 12 weeks of therapy.

Briakinumab is a human anti-IL-12/23 antibody composed of the p40 subunit with human monoclonal IgG1 heavy chain bound to human monoclonal lambda light chain (Table 1). During four phase 3 studies, a PASI 75 response was seen in $81.9 \%, 81.8 \%, 80.7 \%$, and $80.6 \%$ of briakinumab-treated patients at 12 weeks, respectively (Table 1 ). In the phase 2 and 3 extension studies, 99\% of patients showed PASI 75 at 48 weeks, and 76\% showed PASI 100 at week 24. In phase 3 studies, however, five MACE occurred in the group receiving briakinumab versus none in those taking placebo. Twenty-one additional MACE occurred during the phase 2 and 3 extension trials. Because of these events, in July 2011 Abbott withdrew its application for drug approval from the FDA and EMA. This also led to investigation into the relationship between anti-IL-12/23 therapies and vascular inflammation. During the phase 2 and 3 trials 


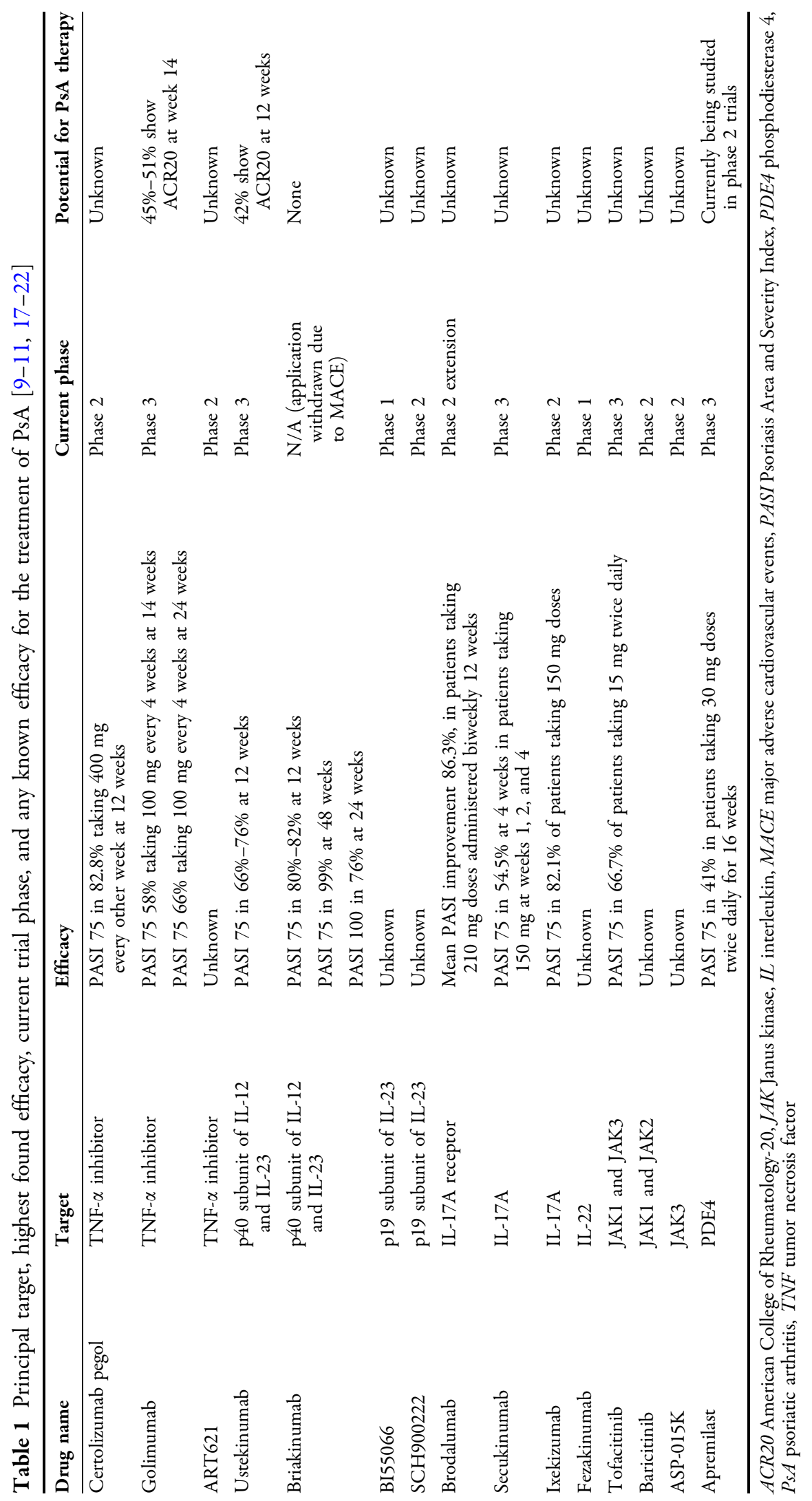


of ustekinumab, 10 MACE occurred compared to zero MACE in the placebo-treated patients [18]. However, further studies investigating the relationship and specific mode of action of these agents relating to coronary artery atherosclerosis and inflammation are still needed to draw conclusions that are more definitive.

More recently, a study showed an increase in RNA expression of the IL-23p19 subunit in psoriatic lesions, but no increase in the expression of the p35 subunit found in IL-12 [16]. These data suggest that IL-23 may be more influential in maintaining psoriatic lesions than IL-12. Furthermore, it has been shown that IL-23 drives keratinocyte proliferation to a greater extent than IL-12 [24]. These observations have led to the development of agents that target the p19 subunit of IL-23. BI655066 (BI) is a humanized IgG1 monoclonal antibody that binds and neutralizes the p19 subunit of IL-23 and is currently in phase 1 trials (Table 1). Merck's (Merck \& Co., Inc, Whitehouse Station, NJ, USA) SCH 900222 is also an anti-IL-23 antibody that targets the p19 subunit. It is currently in phase 2 trials for psoriasis (Table 1) and phase 3 trials are imminent.

\section{INTERLEUKIN-17}

While IL-23 is believed to be a key initiating cytokine in the development and maintenance of Th17 cells and a proven and effective target for psoriasis therapies, inhibiting the Th17 component of the IL-23/Th17 axis has also shown promising results [25]. The innate immune system release cytokines in response to environmental triggers, which leads to activation of myeloid dendritic cells. Myeloid dendritic cells, in turn, secrete IL-23, which

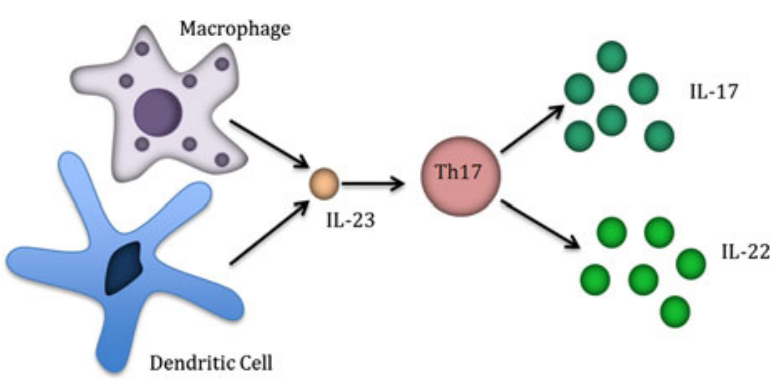

Fig. 1 Relationship between IL-23, Th17 cells, IL-22, and IL-17. IL Interleukin, $T h$ T-helper

drives Th17 differentiation (Fig. 1). Th17 cells produce IL-22 and IL-17, a family of six cytokines (A-F) and five receptors, the levels of which have been shown to be increased in psoriatic skin [26] (Fig. 1). IL-17 is proinflammatory and induces the expression of cytokines, which result in keratinocyte proliferation and epithelial cell inflammation in psoriasis. Increased IL-17 levels lead to an increase in neutrophil migration and survival in the dermis in addition to driving angiogenesis [26].

Brodalumab is a human monoclonal IgG2 antibody that antagonizes the IL-17 pathway by targeting and binding human IL-17A receptor and thus blocking the activity of IL-17A, IL-17F, and IL-17A/F [19]. An initial phase 1 study of $\quad 700 \mathrm{mg}$ brodalumab administered intravenously in 10 patients showed significant clinical improvement at 6 weeks. A phase 2 double-blind, placebo-controlled, dose-ranging study in 198 patients with chronic plaque psoriasis also showed significant improvement at 12 weeks. Patients were randomized to placebo, 70, 140, and $210 \mathrm{mg}$ administered biweekly, or $280 \mathrm{mg}$ administered monthly and were shown to have mean PASI improvements of $16.0 \%$, $45.0 \%, 85.9 \%$, 86.3\%, and $76.0 \%$, respectively [19]. Two cases of neutropenia were reported in the $210 \mathrm{mg}$ brodalumab group. Adverse events included nasopharyngitis, upper respiratory 
tract infection, and injection site erythema [19]. Multiple phase 2 extension studies are ongoing and a phase 3 study comparing safety and efficacy of brodalumab to ustekinumab and placebo is to begin soon.

Secukinumab is a human monoclonal IgG1 antibody that targets the IL-17A cytokine. In an initial proof of concept study in 36 patients, $3 \mathrm{mg} / \mathrm{kg} \quad$ secukinumab administered intravenously demonstrated efficacy at 4 weeks and sustained efficacy at 12 weeks. In an ongoing phase 2 randomized, placebocontrolled trial with active arms, including subcutaneous doses of $150 \mathrm{mg}$ one-time, $150 \mathrm{mg}$ monthly, and $150 \mathrm{mg}$ at weeks 1,2 , and 4, PASI 75 improvements were seen in $12 \%$, $43 \%$, and $54.5 \%$ compared to just $5 \%$ in the placebo arm. A current phase 3 study is ongoing comparing 150 and $300 \mathrm{mg}$ subcutaneous secukinumab doses to placebo. Treatment and placebo groups demonstrated similar safety profiles [20, 27].

Ixekizumab is a human IgG4 monoclonal antibody targeting IL-17A cytokine. In a phase 2 study, 142 patients were randomized to placebo, 10, 25, 75, and $150 \mathrm{mg}$ subcutaneous doses of the ixekizumab [21]. Impressive PASI 75 responses were seen in $76.7 \%, 82.8 \%$, and $82.1 \%$ of patients receiving 25,75 , and $150 \mathrm{mg}$ dosages respectively, compared to only $7.7 \%$ of patients receiving placebo [21]. No serious adverse or cardiovascular events were observed. A phase 3 study comparing safety and efficacy to etanercept and placebo will begin this year.

\section{INTERLEUKIN-20/22}

Although the immunopathogenesis of psoriasis through the Th17/IL-23 axis has been a primary focus for investigators, mechanisms inherent to keratinocyte proliferation and epidermal hyperplasia are also being studied. The IL-10 family of cytokines, specifically IL-20 and IL-22, play an integral role in this process by causing proliferation and altering differentiation of keratinocytes [28]. Additionally, IL-22 expression activates STAT3, which mediates acanthosis, a characteristic histologic finding in psoriasis lesions. Th17 cells secrete IL-22 (Fig. 1), which can induce expression of IL-20 that then acts as a secondary mediator [29]. Thus, the process may also be $\mathrm{T}$ cell driven. These cytokines, which are virtually undetectable in normal, healthy skin, are expressed at increased levels in psoriatic lesions [28]. Elevated levels of IL-22 in the blood have been correlated with disease severity.

Fezakinumab is a humanized monoclonal IgGy antibody to IL-22 being studied for treatment in rheumatoid arthritis and psoriasis. Safety and tolerability results of phase 1 trials for moderate to severe psoriasis are to be published in the near future [11].

\section{JANUS KINASE INHIBITORS}

Cytokines that target STAT transcription factors activate JAK, a type of tyrosine kinase. By inhibiting JAK, the inflammatory action of $\mathrm{T}$ cells and cytokines such as IL-2, -6, -7, -9, -15, and -21 and interferons are suppressed. Tofacitinib is a JAK inhibitor that targets JAK1 and JAK1/JAK3 specifically. It was developed as an immunosuppressant to treat rheumatoid arthritis, ulcerative colitis, Crohn's disease, and psoriasis, and to prevent transplant rejection [30]. In a phase $2 \mathrm{a}$ study of tofacitinib involving 59 patients with psoriasis, twice daily doses of $5,10,20$, and $50 \mathrm{mg}$ and once-daily doses of $60 \mathrm{mg}$ of oral tofacitinib 
were compared versus placebo [11]. After 14 days of treatment, a dose-dependent reduction of Psoriatic Lesion Severity Sum compared to placebo was seen in all patients except those taking the $5 \mathrm{mg}$ dose. Those patients taking $50 \mathrm{mg}$ doses of oral tofacitinib twice daily showed a $71.8 \%$ reduction in PASI (Table 1), whereas the placebo group showed a PASI reduction of only $11.5 \%$. One hundred and ninety-seven patients with psoriasis participated in a subsequent phase $2 \mathrm{~b}$ study, during which subjects were given 2,5 , or $15 \mathrm{mg}$ of oral tofacitinib twice daily [11]. A PASI 75 response was seen in 25\%, 40.8\%, and $66.7 \%$ respectively. Of those treated with placebo, only $2 \%$ achieved a PASI 75 response. Phase 3 studies in psoriasis using up to $10 \mathrm{mg}$ twice daily dosing are currently being performed to confirm safety and efficacy [31] (Table 1).

Baricitinib is an oral drug that selectively inhibits JAK1 and JAK2. During phase 1 studies, baricitinib was shown to be safe and well tolerated in 1-20 mg single doses and repeat doses of $2-20 \mathrm{mg}$. Current phase 2 trials are being performed to determine the efficacy of the drug, the effects of discontinuation, and the safety and efficacy of retreatment (Table 1).

ASP-015K (Astellas Pharma US, Inc., Northbrook, IL, USA) is a JAK 3 inhibitor currently being studied in phase 2 trials [11] (Table 1).

\section{PHOSPHODIESTERASE 4 INHIBITORS}

Through inhibition of PDE4, it is possible to increase the amount of cyclic adenosine monophosphate in leukocytes, minimizing their inflammatory actions such as cytokine transcription, neutrophil degranulation, chemotaxis, and adhesion to endothelial cells.
Therefore, PDE4 inhibitors have the potential to successfully treat immune-mediated disorders such as psoriasis, rheumatoid arthritis, and Behçet's disease. Currently, Celgene (Celgene, Summit, NJ, USA) is developing an oral PDE4 inhibitor, apremilast (CC-10004), to treat psoriasis and psoriatic arthritis (Table 1). When tested on mouse models, histological features of psoriasis and epidermal thickening decreased significantly. Additionally, expression of TNFalpha, intercellular adhesion molecule-1, and human leukocyte antigen-DR in psoriatic lesions were reduced. During a phase 2 clinical trial that involved 260 patients with psoriasis $24.4 \%$ of patients taking $20 \mathrm{mg}$ of apremilast twice daily for 84 days showed a PASI 75 response. Only $10.3 \%$ of those on placebo had the same results [11]. In a subsequent phase $2 b$ study, patients with psoriasis were given 10,20 , or $30 \mathrm{mg}$ doses of apremilast twice daily. PASI 75 was seen in $11 \%, 29 \%$, and $41 \%$, respectively (Table 1). In this study, which involved 352 patients with moderate to severe psoriasis, eight serious adverse events occurred. None of these serious adverse events, however, were thought to be caused by apremilast [22]. Phase 3 trials are now being performed to test the safety and efficacy of two $30 \mathrm{mg}$ doses of apremilast per day for 16 weeks. Apremilast's potential for treatment of palmoplantar psoriasis and psoriatic arthritis are also being researched in phase 2 and phase 3 studies, respectively (Table 1) [11].

\section{CONCLUSION}

Through increased understanding of the immunopathogenesis of psoriasis, major advances are continuing to be made in the realm of new biologic and oral treatments for plaque psoriasis. Specifically, the Th17/IL-23 axis has been a focus for investigators, 
resulting in many novel agents currently in clinical trials. The IL-23p19 inhibitors are of particular interest in targeted therapies as they are present at increased levels in psoriasis skin lesions and believed to contribute less in protecting against opportunistic infections and less likely to be responsible for MACE events than its IL-12 counterpart. Other cytokines such as IL-17 and IL-22 also play important roles in the pathogenesis of psoriasis and agent's inhibition of them has shown promising results [26, 28]. Moreover, oral agents such as JAK kinase and PDE4 inhibitors have proven effective in phase 2 and 3 clinical trials with a different safety profile and may offer options to patients averse to injectable agents. It is important to note, however, these new agents are potentially immunosuppressive and some are already known to increase the risk of serious infections. There is a need to be vigilant for longer-term side effects, including the potential for an increased risk of malignancy, the best method of doing so via established pharmacovigilance registries [32].

Times are promising in the field of targeted therapies for psoriasis, and continued research into the immunopathogenesis of the disease will only lead to a growing armamentarium of safe and effective therapeutic agents.

\section{ACKNOWLEDGMENTS}

Dr. Menter is the guarantor for this article, and takes responsibility for the integrity of the work as a whole.

Conflict of interest. Dr. Alan Menter served on the advisory board of and was a consultant, investigator, and speaker for Abbott labs, Amgen, and Janssen receiving grants and honoraria; served on the advisory board of and was a consultant, investigator, and speaker for Wyeth receiving honoraria; served on the advisory board of and was a consultant and speaker for Galderma, receiving honoraria; served as a consultant and investigator for Stiefel receiving grants and honoraria; served as a consultant and investigator for Eli Lilly receiving grants; served as an investigator for Allergan, Celgene, Novartis, Novo Nordisk, Pfizer, and SyntrixBiosystems receiving grants. Dr. Richard Warren has served as a consultant and/or speaker for Abbott labs, Pfizer, Janssen, Leo Pharma, and MSD.

Open Access. This article is distributed under the terms of the Creative Commons Attribution Noncommercial License which permits any noncommercial use, distribution, and reproduction in any medium, provided the original author(s) and the source are credited.

\section{REFERENCES}

1. Nestle FO, Kaplan DH, Barker J. Psoriasis. N Engl J Med. 2009;36:496-509.

2. Gottlieb A, Korman NJ, Gordon $\mathrm{KB}$, et al. Guidelines of care for the management of psoriasis and psoriatic arthritis: section 2. Psoriatic arthritis with an emphasis on the biologics. J Am Acad Dermatol. 2008;58:851-64.

3. Feldman SR, Fleischer AB, Reboussin DM, et al. The economic impact of psoriasis increases with psoriasis severity. J Am Acad Dermatol. 1997;37:564-9.

4. Gelfand JM, Neimann AL, Shin DB, et al. Risk of myocardial infarction in patients with psoriasis. JAMA. 2006;296:1735-41.

5. Neimann AL, Shin DB, Wang X, et al. Prevalence of cardiovascular risk factors in patients with psoriasis. J Am Acad Dermatol. 2006;558:29-35.

6. Ryan C, Menter AM, Warren RB. The latest advances in pharmacogenetics and pharmacogenomics in the treatment of psoriasis. Mol Diagn Ther. 2010;14:81-93. 
7. Warren RB, Chalmers RJ, Griffiths CE, Menter A. Methotrexate for psoriasis in the era of biological therapy. Clin Exp Dermatol. 2008;33:551-4.

8. Warren RB, Griffiths CE. The future of biologic therapies. Semin Cutan Med Surg. 2010;29:63-6.

9. Ortonne J, Sterry W, Tasset C, Reich K. Safety and efficacy of subcutaneous certolizumabpegol, a new anti-tnf-alpha monoclonal antibody, in patients with moderate-to-severe psoriasis: preliminary results from a double-blind, placebo-controlled trial. In: Annual Meeting of the American Academy of Dermatology; February 2-6, 2007; Washington DC.

10. Kavanaugh A, McInnes I, Mease $\mathrm{P}$, et al. Golimumab, a new human tumor necrosis factor alpha antibody, administered every four weeks as a subcutaneous injection in psoriatic arthritis: Twenty-four-week efficacy and safety results of a randomized, placebo-controlled study. Arthritis Rheum. 2009;60:976-86. Erratum in: Arthritis Rheum. 2010;62:2555.

11. Arana successfully completes Phase II psoriasis study [press release]. New South Wales, Australia: Arana Therapeutics; March 9, 2009. http://www.fiercebiotech.com/press-releases/aranasuccessfully-completes-phase-ii-psoriasis-study. Accessed Jul 252012.

12. Yao Z, Painter SL, Fanslow WC, et al. Human IL-17: a novel cytokine derived from T cells. J Immunol. 1995;155:5483-6.

13. Yawalker N, Tscharner GG, Hunger RE, Hassan AS. Increased expression of IL-12p70 and IL-23 by multiple dendritic call and macrophage subsets in plaque psoriasis. J Dermatol Sci. 2009;54:99-105.

14. Yawalker N, Karlen S, Hunger R, et al. Expression of interleukin-12 is increased in psoriatic skin. J Invest Dermatol. 1998;111:1053-7.

15. Piskin G, Sylva-Steenland RM, Bos JD, Teunissen MB. In vitro and in situ expression of IL-23 by keratinocytes in healthy skin and psoriasis lesions: enhanced expression in psoriasis lesions: enhanced expression in psoriatic skin. J Immunol. 2006; 176:1908-15.

16. Lee E, Trepicchio WL, Oestreicher JL, et al. Increased expression of interleukin 23 p19 and p40 in lesional skin of patients with psoriasis vulgaris. J Exp Med. 2004;199:125-30.

17. Gottlieb A, Menter A, Mendelsohn A, et al. Ustekinumab, a human interleukin 12/23 monoclonal antibody, for psoriatic arthritis: randomized, double-blind, placebo-controlled, crossover trial. Lancet. 2009;373:633-40.

18. Ryan C, Menter A. Psoriasis and cardiovascular disorders. G Ital Dermatol Venereol. 2012; 147:179-87.

19. Papp KA, Leonardi C, Menter A, et al. Brodalumab, an anti-interleukin-17-receptor antibody for psoriasis. N Engl J Med. 2012;366:1181-9.

20. Kurjeza M, Rudnicka L, Olszewska M. New interleukin-23 pathway inhibitors in dermatology: ustekinumab, briakinumab, and secukinumab. Am J Clin Dermatol. 2011;12:113-25.

21. Leonardi C, Matheson R, Zachariae C, et al. Antiinterleukin-17 monoclonal antibody ixekizumab in chronic plaque psoriasis. $\mathrm{N}$ Engl J Med. 2012;366:1190-9.

22. Papp KA, Langley RG, Lebwohl M, et al. Efficacy and safety of ustekinumab a human interleukin-12/ 23 monoclonal antibody, in patients with psoriasis: 52 -week results from a randomized, double-blind, placebo-controlled trial (phoenix 2). Lancet. 2008;371:1675-84.

23. Lebwohl M, Yeilding N, Szapary P, et al. Impact of weight on the efficacy and safety of ustekinumab in patients with moderate to severe psoriasis: rationale for dosing recommendations. J Am Acad Dermatol. 2010;63:571-9.

24. Aggarwal S, Ghilardi N, Xie MH, et al. Interleukin23 promotes a distinct CD4 $\mathrm{T}$ cell activation state characterized by the production of interleukin-17. J Biol Chem. 2003;278:1910-4.

25. Girolomoni G, Mrowietz U, Paul C. Psoriasis: rationale for targeting IL-17. Br J Dermatol. 2012;4:717-24.

26. Cai Y, Fleming C, Yan J. New insights of T cells in the pathogenesis of psoriasis. Cell Mol Immunol. 2012;9:302-9.

27. Hueber W, Patel DD, Drya T, et al. Effects of ain457, a fully human antibody to interleukin-17a, on psoriasis, rheumatoid arthritis, and uveitis. Sci Transl Med. 2010;2:52-72.

28. Wolk K, Witte E, Warszawska K, et al. The Th17 cytokine IL-22 induces IL-20 production in keratinocytes: a novel immunological cascade with potential relevance in psoriasis. Eur J Immunol. 2009;39:3570-81.

29. Tohyama M, Hanakawa Y, Shirakata Y, et al. IL-17 and IL-22 mediate IL-20 subfamily cytokine production in cultured keratinocytes via increased 
IL-22 receptor expression. Eur J Immunol. 2009;39:2779-88.

30. Changelian PS, Flanagan ME, Ball DJ, et al. Prevention of organ allograft rejection by specific janus kinase 3 inhibitor. Science. 2003;302:875-8.

31. Papp KA, Menter A, Strober B, et al. Efficacy and safety of tofacitinib, an oral Janus kinase inhibitor, in the treatment of psoriasis: a Phase $2 b$ randomized placebo-controlled dose-ranging study. Br J Dermatol. 2012;167:668-77.

32. Burden $\mathrm{AD}$, Warren RB, Kleyn $\mathrm{CE}$, et al. BADBIR Study Group. The British Association of Dermatologists' Biologic Interventions Register (BADBIR): design, methodology and objectives. Br J Dermatol. 2012;166:545-54. 